\title{
La prise en charge de la dépendance est-elle un problème de santé ou d'emploi ?
}

Une analyse en termes d'espaces de régulation

\section{Florence Gallois}

\section{(2) OpenEdition \\ Journals}

Édition électronique

URL : http://journals.openedition.org/ei/495

DOI : $10.4000 /$ ei.495

ISSN : 2553-1891

Éditeur

Association Économie et Institutions

Référence électronique

Florence Gallois, «La prise en charge de la dépendance est-elle un problème de santé ou d'emploi ?», Économie et institutions [En ligne], 18-19 | 2012, mis en ligne le 01 février 2013, consulté le 21 avril 2019. URL : http://journals.openedition.org/ei/495 ; DOI : 10.4000/ei.495

Ce document a été généré automatiquement le 21 avril 2019

Revue Économie et institutions 


\title{
La prise en charge de la dépendance est-elle un problème de santé ou d'emploi ?
}

Une analyse en termes d'espaces de régulation

\author{
Florence Gallois
}

\section{NOTE DE L'AUTEUR}

Je remercie vivement les référés anonymes pour leurs critiques constructives sur une version précédente de cet article, je remercie également J-P. Domin et M. Nieddu pour leurs commentaires sur différentes versions antérieures de ce travail. Je reste seule responsable de ses erreurs et insuffisances

1 L'aide à domicile aux personnes âgées dépendantes relève d'une catégorie institutionnelle de services, les services à la personne, créée par le Plan Borloo (2005) à partir de deux grands secteurs aux trajectoires historiquement distinctes: des services sociaux et des services de domesticité . À des fins de créations d'emplois, le Plan Borloo cherche à soutenir l'émergence d'un nouveau secteur par l'introduction de dispositifs institutionnels supposés les unifier. Cependant, des filières économiques et de production rattachant différentes activités des services à la personne à des systèmes extérieurs persistent, notamment l'aide à domicile peut être rattachée aux filières de santé. La volonté politique de recomposition d'un ensemble initialement distinct d'activités en un secteur soulève donc un problème de définition et de frontières de l'espace de régulation ${ }^{1}$ , que l'on se propose ici d'analyser sous l'angle particulier de la prise en charge de la dépendance par le biais des services d'aide à domicile.

2 La représentation de la protection sociale proposée par B. Théret sous forme d'un système composé des ordres économique, politique et domestique, dont l'unité et la cohérence doivent être interprétées, permet d'éclairer ce qui se joue dans la prise en charge de la dépendance. En effet, la prise en charge de la dépendance constitue une 
forme d'impératif social, au sens où, quoi qu'il arrive, il faudra bien que la prise en charge ait lieu, que ce soit dans l'ordre politique, économique ou domestique.

3 La dépendance est le plus souvent associée à un état pluripathologique de la personne, qui apparaît à la suite d'un problème d'ordre médical. Bien que distincts, dépendance et maladie constituent ainsi deux phénomènes à fortes interactions chez les personnes avancées en âge . Or, si l'on considère que "la santé est un état complet de bien-être et ne consiste pas seulement en une absence de maladie ou d'infirmité", il devient évident que le corps médical n'a pas le monopole de la santé, mais que nombre d'autres acteurs y concourent. Parmi ceux-ci, les structures d'aide à domicile (SAD) contribuent à la prévention d'une dégradation de l'état de santé (p. ex. suivi de l'alimentation) et jouent un rôle de veille sanitaire. Ainsi positionnée, il est légitime de considérer que l'aide à domicile est partie intégrante du système de santé.

4 Mais, exclue du champ de l'assurance maladie, l'aide à domicile ne relève pas du secteur du soin et n'est, de ce fait, pas reconnue comme un acteur de santé à part entière, même si, nous le verrons dans la suite de ce travail, en offrant une possibilité de substitution (au moins partielle) avec d'autres formes de prise en charge, elle joue un rôle majeur dans la soutenabilité des politiques de santé.

5 L'aide à domicile peut être rattachée à un autre espace de régulation : celui des services à la personne. Ces services comprennent un ensemble d'activités hétéroclites ${ }^{2}$, unifiées légalement en un même secteur par le Plan Borloo et les dispositifs qu'il a introduits. Avec un objectif annoncé de créer 300000 emplois en trois ans, le Plan vise à soutenir la création d'un marché des services à la personne. Ce qui peut apparaître paradoxal, dans ce schéma, est que l'État se positionne de façon interventionniste afin de faire émerger de nouveaux comportements de consommation (supposés répondre à des besoins latents). En cette optique, un ensemble de mesures visant à générer une baisse du coût d'opportunité pour les consommateurs potentiels est mis en œuvre (en particulier, une réduction d'impôt à hauteur de $50 \%$ des sommes engagées). La puissance publique intervient au nom de la dimension sociale de traitement du chômage, et ce alors que son intervention, dans l'espace ainsi délimité, était, historiquement, essentiellement liée à d'autres dispositifs relevant de la protection sociale et de l'aide sociale destinés à soutenir l'aide à domicile.

6 L'aide à domicile se situe donc au chevauchement du système de santé et des services à la personne. À ce croisement, des logiques d'articulation avec la protection sociale, si ce n'est réellement opposées, à tout le moins disparates coexistent et rendent la régulation du sous-secteur de l'aide à domicile particulièrement confuse.

7 À partir d'une démarche abductive, nous cherchons à montrer un manque de cohérence dans la régulation de l'aide à domicile. L'enchevêtrement de régulations relevant de logiques différentes est une question centrale dans la littérature du champ. Ainsi, les travaux d'Enjolras , d'Enjolras et Laville ou, plus récemment, ceux de Gardin, Nyssens et Minguizzi ainsi que Fraisse et Gardin pointent d'une part, l'introduction de mécanismes marchands dans la régulation de services sociaux, et d'autre part un enchevêtrement entre les logiques d'emploi attribuées à l'aide à domicile par la puissance publique et la logique sociale de la prise en charge de personnes au moyen de l'aide à domicile. Notre démarche diffère dans la mesure où nous positionnons l'aide à domicile comme un service de santé et non seulement un service social. 
8 La régulation des services d'aide à domicile est alors examinée dans un premier temps au prisme de la santé. Nous positionnons l'aide à domicile au sein d'un espace de régulation potentiel : le système de santé. Le recours à une analyse sur longue période du système de soins et de son organisation permet de soulever comment le passage d'un système hospitalo-centré à un système favorisant les soins de ville a conduit à l'émergence d'offres de soins alternatives et a rendu nécessaire de positionner l'aide à domicile comme élément du système de santé(1). Dans un second temps, nous interrogeons le rapport à la protection sociale des activités d'aide à domicile, considérées au regard de leur inscription au sein de l'espace des services à la personne (2).

\section{L'aide à domicile : fonction indispensable à la soutenabilité de la réorganisation de l'hôpital}

9 Initialement lieu de charité et d'accueil des plus démunis, l'hôpital s'est progressivement imposé, depuis les années 1950, comme élément central du système de santé. Son développement a été accompagné par celui du système de protection sociale et de l'accès aux soins. Toutefois, les progrès de la médecine et l'introduction de logiques marchandes, à partir des années 1980, ont conduit l'hôpital à recentrer ses missions sur la prise en charge de la maladie aiguë et ont amené à une réduction majeure des durées moyennes de séjour (DMS) (1.1). Le système de santé se réorganise et des relais de soins deviennent nécessaires, ce qui conduit au positionnement de l'aide à domicile comme acteur de la filière (1.2). Mais les services d'aide à domicile restent à une frontière mal définie du système de santé, dont les évolutions du financement de la dépendance indépendamment de la maladie témoignent (1.3).

\subsection{Marchandisation, maîtrise des dépenses et baisse des durées de séjour}

10 L'introduction de logiques de productivité et d'éléments marchands dans le système de santé repose grandement sur l'évolution des modalités de financement de l'hôpital. La première réforme est le passage, en 1983, du système du prix de journée, jugé inflationniste et incitant au remplissage des lits ${ }^{3}$, au détriment de la réduction des durées de séjours, à un système de budget global. Cette forme de rémunération va introduire des logiques productivistes. Le montant de la dotation de l'hôpital y est déterminé à partir d'un budget prévisionnel. Le budget est ensuite négocié (du moins en théorie) avec l'État après avis de la $\mathrm{CRAM}^{4}$. Mais dans les faits, l'État va davantage imposer le budget que mener une discussion avec l'établissement : le poste des rémunérations est décidé par la tutelle, les investissements sont soumis à des contraintes strictes et les taux directeurs (instaurés en 1979), qui conditionnent l'évolution des budgets, opèrent un rationnement des établissements. Ces nouvelles modalités de tarification, déconnectées de l'activité de soins et instaurant une contrainte budgétaire forte sur les établissements, ont eu des conséquences négatives, dénoncées comme étant des «effets pervers incompatibles avec les missions du service public ». L'hôpital passe d'une logique de production à une logique de productivité, les créations de postes sont opérées à budgets constants. Dans la mesure où le système du budget global n'incite pas à prolonger la durée de séjour dans un but d'accroissement des ressources, il n'est pas étonnant de voire la contrainte financière, combinée aux progrès de la médecine, peser sur la durée de séjour : la durée moyenne de 
séjour (DMS) des disciplines de courts séjours (Médecine, Chirurgie et Obstétrique - MCO) diminue de plus de $34 \%$ entre 1983 et 1996 (cf. Figure 1). En soins de suite et de réadaptation (SSR), elle diminue de $30 \%$.

Considéré comme un étau financier qui freine l'activité de soins, le budget global est remis en question par l'ensemble du corps hospitalier dès la fin des années 1980. L'idée d'une tarification à la pathologie, donc à partir de l'activité de soins, émerge.

Figure 1 - Évolution des durées moyennes de séjour

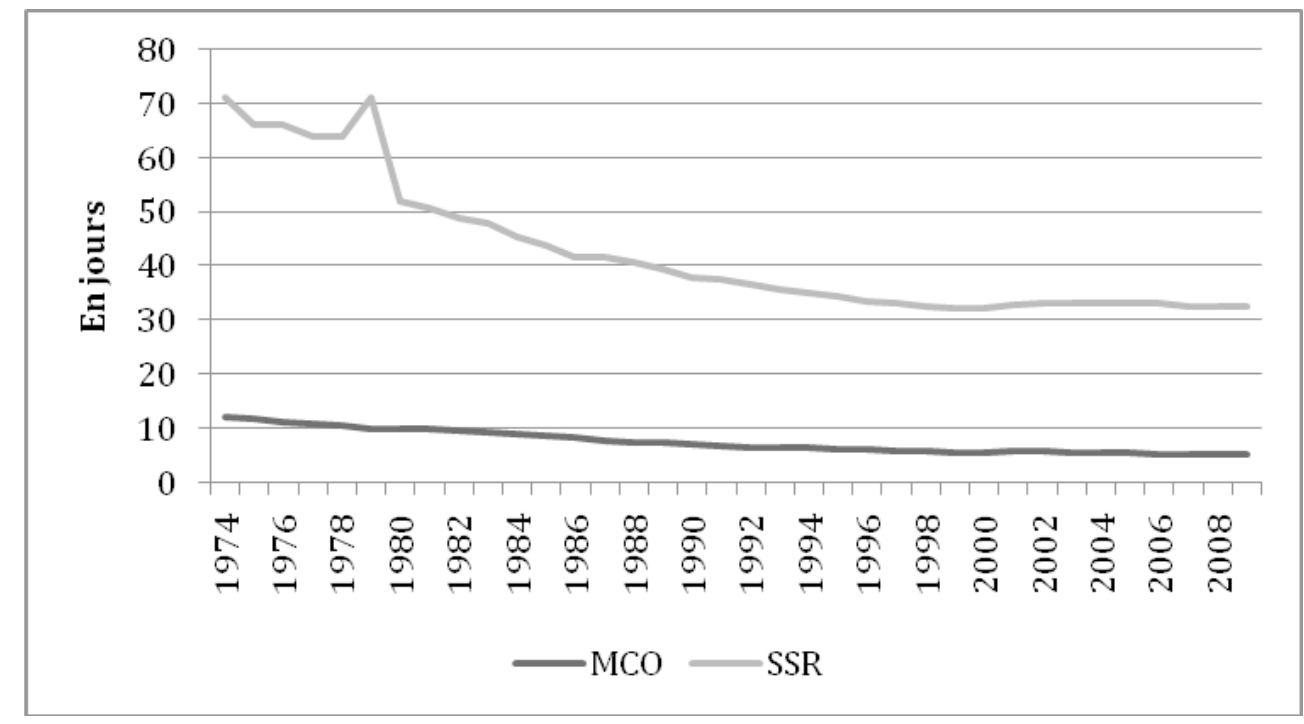

Source : DREES, base Eco-santé France 2011 (mise à jour juillet 2011)

En 1996, les recettes de l'hôpital se verront pondérées en fonction de son activité. L'instauration de cette tarification, fonction de l'activité médicale, marquera le passage d'une régulation tutélaire à une régulation quasi marchande, dont l'avènement sera la mise en place de la tarification à l'activité (T2A) en 2004. L'hôpital est désormais rémunéré à l'acte pour les activités de $\mathrm{MCO}$. L'objectif prioritaire de cette nouvelle réforme est la performance économique, obtenue par une réduction des coûts de production et un accroissement de la productivité . Le prix étant fixé indépendamment du coût réel, l'établissement se voit incité à diminuer ses coûts de production pour être rentable au regard du prix d'achat . L'obtention de cette performance passe notamment par une amélioration du taux de rotation des ressources engagées et in fine une réduction de la durée de séjour conduisant à un report sur d'autres formes de prise en charge et sur la médecine de ville.

Il n'existe pas, à notre connaissance, d'analyse des transferts de patients d'un mode de prise en charge à un autre qui seraient induits par la T2A. Néanmoins, l'engouement affiché pour la rentabilité de la chirurgie ambulatoire et en faveur du développement de l'hospitalisation de jour, en partie pour des raisons similaires, indique que cette modalité de tarification constitue bien un outil économique en faveur du raccourcissement de la durée des séjours. Ce système inquiète toutefois certains médecins quant aux possibilités d'offrir une prise en charge globale du patient quand la rémunération de l'hôpital s'organise autour de séjours courts à objectif de soins unique et au risque induit «de faire coïncider la durée du séjour avec la borne basse [...], au risque d'hospitalisations itératives ». Ces professionnels sont d'autant plus inquiets que les études menées aux États-Unis ont mis 
en évidence que la baisse des durées de séjour était corrélée à un accroissement significatif (et que Rouget qualifie de "démesuré ») des soins de suite et de réadaptation (SSR) et des soins à domicile, traduisant un système de vase communiquant entre plusieurs acteurs de la filière de soins. Ce phénomène de transfert n'est d'ailleurs pas ignoré des tutelles françaises qui soutiennent, depuis le début des années 2000, la création de places de soins en dehors du court séjour, notamment en hospitalisation à domicile et en soins de suite et réadaptation .

L'examen de l'évolution des modalités de financement de l'hôpital soulève que, dès l'instauration du budget global, la contrainte budgétaire faisait pression sur la durée de séjour. En outre, cette pression a eu tendance à s'accentuer avec l'introduction de la tarification à l'activité. L'hôpital a été incité à se centrer sur la phase aiguë de la maladie, dont la prise en charge nécessite des investissements lourds (plateau technique). Cela s'est traduit par une diminution franche des durées de séjour. En court séjour (MCO), la durée moyenne de séjour est passée de plus de 12 jours en 1974 à 5,2 en 2009, soit une réduction de $57 \%$ en 25 ans.

Si les progrès de la médecine peuvent, dans une certaine mesure, contribuer à réduire la durée de prise en charge, cette raison ne peut être la seule invoquée et il est apparu au cours de notre exposé que le mode de tarification de l'hôpital exerce également une influence. La T2A, en incitant à diminuer les coûts de production, conduit à raccourcir les durées de séjour. Il devient donc nécessaire d'organiser une offre de relais de soins par des services d'hospitalisation à domicile, de soins infirmiers à domicile et d'aide à domicile, et de coordonner ces services autour du patient.

\subsection{Organisation d'une offre alternative, entre substituabilité et complémentarité}

Le repositionnement de l'hôpital dans le système de santé va s'accompagner de l'émergence d'une offre alternative. La prise en charge des patients à leur domicile, par des services d'Hospitalisation à domicile (HAD) et par des Services de soins infirmiers à domicile (SSIAD), constitue une solution crédible et souhaitable pour les hôpitaux, notamment pour les patients n'étant pas à un stade aigu de la maladie. Cette offre, au sein de laquelle les charges d'hôtellerie (repas, linge) sont transférées sur l'entourage du patient, permet à l'Assurance maladie de réaliser des économies relativement aux hospitalisations à temps complet tout en assurant des soins de même qualité .

Dans ce contexte, les fédérations associatives du champ médico-social militent en faveur de l'émergence de solutions complémentaires à l'hospitalisation . Les alternatives à l'hospitalisation qui seront développées le seront cependant davantage dans une optique de substitution de l'hôpital par le médico-social. Ainsi, sous l'impulsion de la loi portant réforme hospitalière du 31 juillet 1991, l'hôpital se réorganise et diminue le nombre de places de court séjour par le biais de fermetures ou de reconversions, d'autres modalités de prise en charge des malades sont amenées à se développer telles que la création de place de SSIAD et de places en section de cure médicale. On assiste alors à un transfert des patients (en particulier des patients âgés) de l'hôpital vers d'autres modalités de prises en charge en maison de retraite et à domicile.

La reconversion de l'offre hospitalière de long séjour relève de cette logique de substituabilité. De façon surprenante, c'est dans une loi organisant la prise en charge de 
la dépendance (instauration de la Prestation spécifique dépendance - PSD - en 1997) que les critères de substitution entre les deux offres sont définis : l'hospitalisation en long séjour est destinée à des personnes nécessitant des soins prolongés ayant un caractère très médicalisé et nécessitant un plateau technique ; par opposition les autres personnes relèvent de l'établissement médico-social. Or, sur les 80000 lits existants lors de la mise en œuvre de cette mesure, 97 \% sont occupés par des personnes âgées dépendantes, dont le ministère estime qu'elles relèvent du médico-social . De fait, les établissements hospitaliers de long séjour sont incités à se reconvertir en établissement médico-social. Des frontières du système se redessinent au sein même de celui-ci avec un recloisonnement des modalités (financières) de prise en charge.

Le processus de diminution du nombre de places à l'hôpital, et de transfert des malades et personnes dépendantes vers le social et le médico-social remonte au début des années 1980 , lors de la mise en œuvre de mesures incitant à des logiques productivistes. On observe ainsi sur la décennie une diminution d'ensemble de 7,5\% du nombre total de lits d'hospitalisation et de $12 \%$ pour les seuls MCO (cf. Figure 2). Dans les années 1990, le phénomène s'intensifie : le nombre de places total diminue de $12 \%$, celui de MCO de plus de $15 \%$ et les Soins de suite et de réadaptation (SSR) de $5 \%$, tandis que le nombre de places en Soins de longue durée (SLD) s'accroît de 23 \%. À partir de 1993, date à laquelle nous disposons de données sur le médico-social, il est possible de mettre en balance la diminution du nombre de lits d'hôpital et le développement de solutions médico-sociales alternatives. Ainsi, là où la capacité totale de l'hôpital diminue de près de $10 \%$, le nombre de places de SSIAD croît de plus de $33 \%$, sans pour autant compenser en valeur absolue les fermetures de places $(-84700$ contre +15800$)$. Le nombre de places en établissement d'hébergement pour personnes âgées s'accroit de plus de $30 \%$ (représentant plus de 56000 nouvelles places).

Les années 2000 suivent la même tendance: entre 2000 et 2008, le nombre de lits d'hospitalisation diminue de près de $12 \%$, avec une réduction particulièrement forte du nombre de lits en soins de longue durée (- $43 \%$ ), attribuable à une reconversion en établissement médico-social pour l'accueil de personnes âgées. Se développe également le système de l'hospitalisation à domicile dont la capacité d'accueil va plus que doubler entre 2000 et 2009, passant de 5364 lits à 12 273. Néanmoins, les capacités créées en HAD ne compensent pas les fermetures de lits au sein de l'hôpital, et il est communément admis que les SSIAD sont amenés à compenser les défaillances de l'offre d'HAD en prenant en charge des patients qui devraient être hospitalisés à leur domicile compte tenu de leur état de santé et de leurs besoins de soins . 
Figure 2 - Évolution du nombre de lits et places à l'hôpital et dans les solutions alternatives

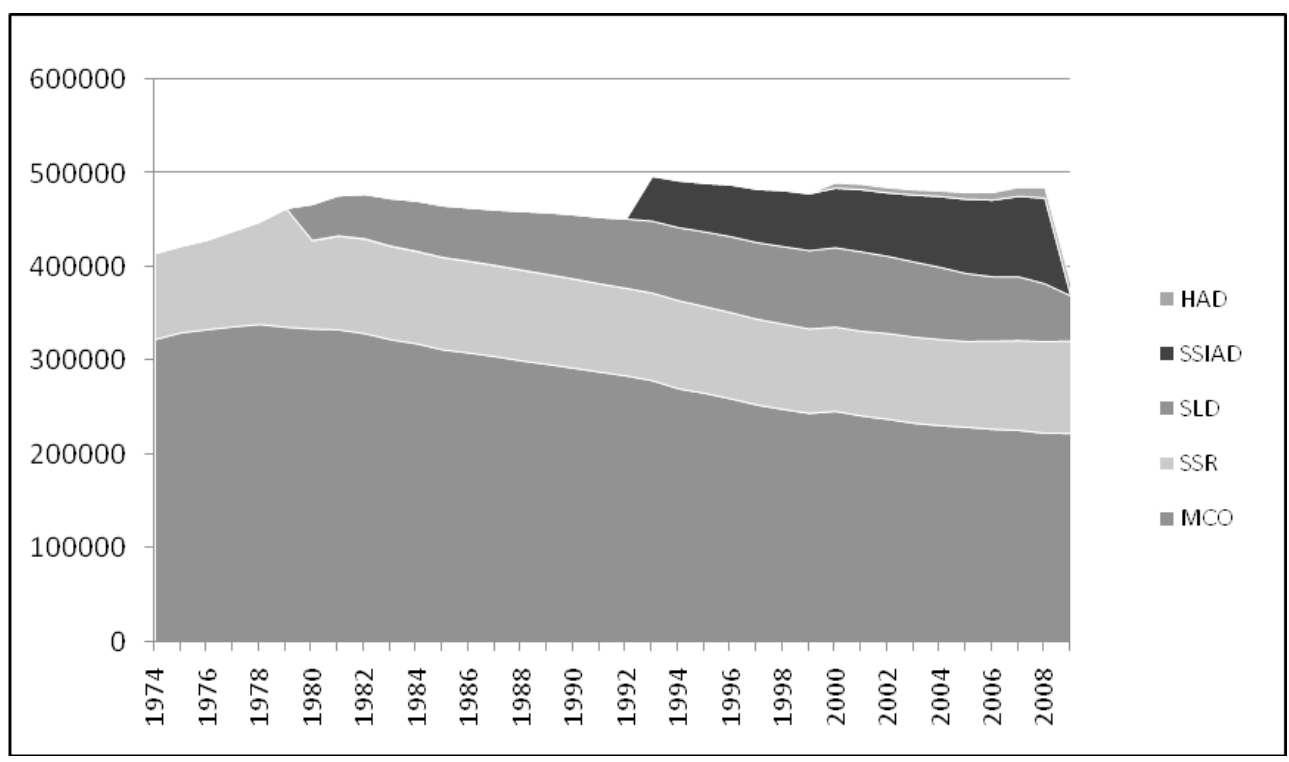

Source : DREES, base Eco-santé France 2011 (mise à jour juillet 2011)

21 Mais malgré de fortes créations, les taux d'équipement en SSIAD restent relativement stables, ils ont évolué de 15 à 17 places pour 1000 habitants de plus de 75 ans entre 1993 et 2008, alors que les besoins sont estimés à 30 places . La conséquence de ce rationnement de l'offre de SSIAD est la création (le maintien) de listes d'attente pour la prise en charge des patients . Ceux-ci sont alors transférés sur les services d'aide à domicile dont l'offre n'est pas contingentée ${ }^{5}$. Les SAD interviennent également (et bien souvent dans l'urgence) pour soutenir (au sens de rendre possible) des sorties d'hôpital de personnes non autonomes.

Le taux d'équipement en établissements d'hébergement médicalisé pour personnes âgées, qui était de 53,4 places pour 1000 habitants de plus de 75 ans en 1993, a lui connu une croissance de $7 \%$ entre 1993 et 2000 et plus que doublé sur la période 1993-2008, mais l'incohérence des données ${ }^{6}$, en particulier les ruptures constatées à partir de 2000, nous fait considérer ce chiffre avec la plus grande prudence. De la même manière que l'on observe un transfert de l'HAD aux SSIAD puis sur l'aide à domicile, l'existence de files d'attente dans les Établissements d'hébergement pour personnes âgées dépendantes (EHPAD) conduit à un transfert sur les services d'aide à domicile.

23 Le développement de formes alternatives de prise en charge, qui viennent en partie substituer l'hôpital, traduit une logique de rationalisation des soins. Celle-ci organise une recomposition $\mathrm{du}$ système de santé et de ses frontières. Elle s'opère par un repositionnement de la personne, malade ou dépendante, au centre d'un processus de soins faisant désormais intervenir une multitude d'acteurs. La coordination de ces acteurs est partiellement organisée par les tutelles à travers les schémas sanitaires et sociaux, mais d'autres formes de coordination se mettent en œuvre : les réseaux. Les économistes de la santé distinguent dans le champ de la santé deux formes de réseaux: le réseau comme forme d'allocation de ressources, inscrit dans une perspective de régulation marchande et le réseau comme forme de création de ressources où la régulation s'appuie sur la coordination des acteurs. Néanmoins, seule cette dernière forme de réseau comme forme d'organisation de ressources complémentaires dans la prise en charge peut être 
observée dans l'aide à domicile. Elle joue un rôle essentiel à la réalisation de la relation de service : $43 \%$ des recours à l'aide à domicile découlent de l'intervention de professionnels des soins ou du social, qui jouent un rôle d'intermédiaire entre les prestataires de services et les bénéficiaires (la famille et la réputation des structures d'aide à domicile constituant les autres intermédiaires) . L'intermédiation se rattache à plusieurs types de réseaux, qui coexistent. On dénombre ainsi des réseaux/formes de coordination entre médecine de ville et hôpital qui permettent le développement de l'hospitalisation à domicile. On trouve également des réseaux de santé, composés principalement de soignants, traitant des problèmes spécifiques des patients âgés. Enfin, portés davantage par les acteurs sociaux et adoptant une vision globale des besoins des personnes âgées (comprenant à la fois le logement, l'aide à la vie quotidienne et la santé), les réseaux de coordination gérontologiques (CLIC). Ainsi, même si les acteurs de la prise en charge sont amenés à se coordonner, une séparation persiste au point de vue de l'organisation formelle des réseaux, les réseaux de type social étant distincts de ceux de la santé.

Le phénomène de transfert des patients de l'hôpital vers des modes de prises en charge relevant du social et du médico-social vise l'efficience de l'utilisation des ressources hospitalières et des ressources finançant les soins (en particulier la phase aiguë) tout en visant la mise en œuvre de prises en charge mieux adaptées aux besoins des patients. De ce point de vue, il ne peut qu'être soutenu. Toutefois, il induit un transfert des patients de financements rattachés à l'Assurance maladie vers des modalités de prises en charge de la dépendance complexes et historiquement distinctes de la santé, structurée, elle, autour du soin et de la maladie.

\subsection{Au sein du système de santé : financement de la dépendance et contours de la protection sociale en questions}

La santé est reconnue comme un risque de protection sociale et bénéficie à ce titre de financements socialisés, alloués selon le besoin de consommation de soins et non selon les revenus. Ces financements sont gérés par une instance nationale, la CNAMts qui agit grandement sur le régime économique de fonctionnement du secteur : l'offre se voit ainsi régulée par la fixation des tarifs des soins, les conventions des médecins généralistes... la demande, quant à elle, est conditionnée par l'Assurance maladie obligatoire de la Sécurité sociale. Les financements issus de la Sécurité sociale représentaient près de $76 \%$ des dépenses liées à la Consommation de santé et de biens médicaux (CSBM) en 2010 . Ils sont complétés par une part croissante issue des assurances maladies complémentaires (13,5\% ) et de la contribution directe des ménages (le reste à charge est légèrement supérieur à $9 \%$ en 2010).

Bien que fortement revendiqué par les acteurs du champ social et médico-social, ce trait structurant de la régulation de la santé ne se retrouve pas pour la prise en charge des personnes dépendantes, en particulier dans le cas qui nous intéresse de l'aide à domicile. On assiste au contraire à une succession et une instabilité de dispositifs de financements socialisés, aux limites régulièrement dénoncées. Les débats et évolutions récurrentes des modalités de financement nous semblent révélateurs des problèmes posés par la nonplace des services d'aide à domicile dans la Sécurité sociale alors même que leur activité contribue à la soutenabilité du système de protection sociale, en particulier du système de santé. 
27 La question du financement de la prise en charge de la dépendance s'est posée dès les prémices des modes de prises en charges en dehors de l'hôpital et en dehors du cercle familial; le développement de l'aide à domicile en tant que services permettant le maintien à domicile de personnes dépendantes, étant largement conditionné à l'existence de financements socialisés et à leur périmètre. Les premiers arrangements, trouvés dans les années 1960 et 1970, ne positionnaient pas l'aide à domicile dans le champ de la santé : ils reposaient sur une mise en œuvre fragmentée et facultative par les caisses de retraite à travers leurs crédits d'action sociale. Mais cette organisation tutélaire a conduit à un rationnement de l'offre des prestataires, rapidement insuffisante pour faire face à la demande et aux besoins. En conséquence, une partie de la demande s'est reportée sur une autre forme de production d'aide à domicile : l'emploi direct d'une aide à domicile par la personne âgée, encouragé dès 1973 par l'introduction d'exonérations spécifiques de cotisations sociales pour lesquelles l'éligibilité est fonction de l'état de santé ${ }^{7}$, mais ne bénéficiant pas d'autres formes de financements socialisés.

Le premier dispositif de subventionnement direct du recours à l'aide à domicile est introduit en $1975^{8}$. Il s'agit de l'Allocation compensatrice pour tierce personne ${ }^{9}$ (ACTP), ouverte aux personnes nécessitant l'aide d'un tiers, qu'elles soient âgées ou handicapées. Une distinction sera progressivement opérée entre ces deux types de publics, avec l'introduction en 1987 d'exonérations de cotisations sociales destinées aux personnes âgées (et non aux seules personnes souffrant d'une perte d'autonomie) et l'attribution de nouveaux droits spécifiques aux personnes handicapées.

29 Le système de l'emploi direct se voit ainsi encouragé alors que cette forme de production ne bénéficie pas d'un encadrement par des tutelles et n'apporte aucune garantie objective de qualité de service (continuité des interventions, etc.). Son organisation fait au contraire référence à l'emploi domestique non qualifié et subordonné à des relations personnelles , éloigné de fait d'une logique de santé dispensée par des professionnels.

De façon paradoxale, les producteurs d'aide à domicile vont pourtant développer une forme d'offre qui repose sur les dispositifs favorisant les particuliers-employeurs . Il s'agit du mandataire, initié au début des années 1980, où une association apporte un appui à un particulier pour les fonctions liées à son rôle d'employeur. L'émergence de ce système sera encouragée par des dispositifs en faveur de l'emploi qui vont positionner l'aide à domicile dans le champ des politiques d'emploi tout en maintenant une confusion entre services de care et domesticité. Son développement implique en effet une évolution des institutions de l'emploi afin de permettre l'existence légale de ce type de services. Le point saillant de cette évolution est l'officialisation de la capacité des associations d'aide à domicile à servir d'intermédiaire d'emploi. Cela passe par la remise en cause, en 1986, du monopole de l'ANPE quant au placement des demandeurs d'emploi. Le soutien public à cette forme de production d'aide à domicile apparait alors comme une politique d'emploi dont l'objectif est d'améliorer l'efficacité des marchés du travail via une plus grande flexibilité. Ce faisant, elle positionne les associations d'aide à domicile (et surtout les associations mandataires) comme acteurs à part entière de la mise en œuvre des politiques d'emploi mais les éloigne de la santé et de la Sécurité sociale. Le développement de l'aide à domicile ne peut dès lors être détaché de politiques d'emplois portées et mises en œuvre par les associations d'aide à domicile.

31 À la fin des années 1980, la fédération UNASAD (devenue UNA) cherche pourtant à inscrire l'aide à domicile dans une autre logique, celle de la santé. Elle milite en faveur de la création d'un cinquième risque de Sécurité sociale. Au regard de ses conséquences, la 
dépendance constitue un risque social: sa survenue conduit à un surcroît de dépenses pouvant être accompagné d'une diminution de revenus (si la personne est en emploi lorsque survient l'incapacité). La promotion de ce nouveau risque s'oppose à une couverture départementale, qui renvoie à l'image d'action sociale et d'assistance. Le rattachement à la Sécurité sociale nationale, financée par cotisations, offre des perspectives de plus grande pérennité et stabilité du financement de la prise en charge de la dépendance relativement à une prise en charge départementale, financée par l'impôt, et pouvant faire l'objet de coupes budgétaires.

Dans les premiers temps du débat, la question de l'intégration de la dépendance au risque santé est posée, mais l'option est rapidement écartée. En effet, au milieu des années 1990, le problème de la dépendance est posé comme un problème de santé. La conférence nationale de santé (1996) a considéré comme prioritaire le problème de la dépendance et souhaité la création d'une allocation pour les personnes âgées dépendantes, mais n'a pas tranché sur son financement, ne souhaitant pas se positionner entre une allocation d'aide sociale et une prestation relevant de l'Assurance maladie. Un financement par les Conseils généraux (dont les compétences en action sociale ont été affirmées par la loi de décentralisation du 2 mars 1982), appuyés par des ressources spécifiques de l'État va progressivement s'imposer. Ce système, déjà en œuvre avec les expérimentations de 1994 va perdurer avec la prestation spécifique dépendance (PSD) instaurée en 1997 et sera réaffirmé lors de la mise en place de l'allocation d'autonomie (APA) en 2001.

La prise en charge de la dépendance organisée par les pouvoirs publics se fait pourtant dans une logique éloignée de la santé et de son financement par la Sécurité sociale. Elle opère une segmentation selon l'âge et l'attribution d'allocations issues de financements socialisés se fait en fonction des revenus de la personne dépendante et non de ses seuls besoins. Ainsi la PSD rompt le principe d'universalité qui prévaut en santé en étant conditionnée non seulement à l'âge et l'état de santé, mais également aux revenus ${ }^{10}$. Audelà, elle organise des distorsions de concurrence en faveur de l'emploi direct qui est également la cible d'une politique d'emploi à destination des non-qualifiés. Et c'est là une distinction majeure avec la prise en charge de la maladie pour laquelle d'une part le remboursement de l'acte ne peut avoir lieu que si le professionnel est reconnu comme tel (nécessité de diplôme d'État, exclusion des formes de médecines alternatives) et où la concurrence est régulée dans cette logique professionnelle.

La mise en œuvre de l'APA, en 2001, permettra de corriger certains défauts de la PSD. Le champ de l'APA est pensé comme universel (le revenu n'est plus une condition d'éligibilité, mais va déterminer la participation, la récupération sur patrimoine est supprimée), ses conditions d'attribution sont prévues pour être similaires à l'ensemble du territoire. Le dispositif opère une hybridation des financements qui positionne la prestation entre aide sociale et Sécurité sociale. Le dispositif APA à domicile préconise, pour les personnes les plus dépendantes, l'intervention de services prestataires et désincite à l'emploi direct en instaurant une majoration du reste à charge du bénéficiaire pour ce type de recours. Les biais concurrentiels introduits par la PSD sont ainsi réduits. À sa mise en œuvre, l'APA laisse entrevoir au champ de la dépendance les conditions nécessaires à un rapprochement avec la maladie de par la perspective d'intégration de la dépendance au sein de la Sécurité sociale et par l'introduction de mécanismes supposés favoriser l'émergence et la reconnaissance d'une profession. L'insertion de l'aide à domicile dans le champ de l'action sociale et médico-sociale par la loi 2002-2 ${ }^{11}$ va poursuivre le mouvement de reconnaissance de l'aide à domicile comme profession. Le 
cadre de l'autorisation médico-sociale, et de la tarification qui lui est associée, constitue, pour les organismes d'aide à domicile, un encouragement à la professionnalisation ainsi qu'une reconnaissance de leur statut de service social exerçant des missions d'intérêt général.

Le mouvement de rapprochement de l'aide à domicile vers le médico-social et la santé sera cependant rapidement inversé. Le Plan Borloo de 2005 affiche clairement les services à la personne, y compris l'aide à domicile, comme un espace où peuvent être créés de nombreux emplois non qualifiés à condition que la puissance publique soutienne l'émergence d'un marché. Il est donc institutionnellement reconnu que les services d'aide à domicile autorisés sont en concurrence entre eux, mais aussi avec l'emploi direct, aux coûts moindres mais sans exigence de qualité, ainsi qu'avec des entreprises peu engagées dans la professionnalisation de leurs salariés. Ceci a des implications directes en termes de financement des services autorisés et indirectes au regard du financement de la dépendance. La procédure de tarification des organismes autorisés organise une concurrence par comparaison, où le tarificateur va pouvoir mimer le marché, la concurrence renforcée par le Plan Borloo pouvant encourager le tarificateur à ne plus intégrer au tarif certaines dimensions des services d'aide à domicile produits par les prestataires associatifs ${ }^{12}$. Or, le Conseil général est à la fois tarificateur et le financeur direct de l'APA, si bien que la non intégration de certaines dimensions du service d'aide à domicile dans la tarification revient à exclure ces dimensions du financement de la dépendance. Par les stratégies différenciées localement différenciées qu'ils développement, ces régulateurs peuvent soit en privilégier une dimension d'action sociale, que nous associons à la santé, soit une dimension d'emplois associée à une moindre qualité . Mais en tout état de cause, la saturation des plans d'aide pour les personnes ayant les besoins les plus importants, compte tenu de l'existence de plafonnements de l'allocation, conduit vers les formes les moins onéreuses et donc, le recours à l'emploi direct, renouvelant la confusion autour de l'aide à domicile, qui la place à la fois comme un élément du système de santé que celui-ci ne finance pas, et comme la cible d'une politique d'emploi.

36 Au regard des dispositifs institutionnels, la Loi portant réforme de l'Hôpital et relative aux patients, à la santé et aux territoires (dite loi HPST ${ }^{13}$ ) entérine l'exclusion de l'aide à domicile aux personnes âgées du champ de la santé : celle-ci n'est en effet pas incluse dans le champ d'action de la Loi alors que tous les autres établissements et services socio et médico-sociaux reconnus par la loi 2002-2 le sont. Cela est d'autant plus paradoxal que la performance et la soutenabilité d'un système de santé, réorganisé autour de prises en charges hospitalières de courte durée, est soutenue par l'existence de services d'aide à domicile.

Au global, l'instabilité et la complexité des dispositifs de financements (et plus largement de régulation) de l'aide à domicile traduisent bien son exclusion relative du système. Cette exclusion relative traduit un problème de positionnement des services d'aide à domicile au sein de la protection sociale. La non-soutenabilité récurrente des dispositifs de financements conduit les associations d'aide à domicile à travailler les contours de la Sécurité sociale afin de s'insérer dans des risques déjà existants (vieillesse à l'origine, maladie par la suite, mais aussi chômage) ou de tenter d'en créer un nouveau: le cinquième risque dépendance. La non-prise de décision publique sur la place réelle de l'aide à domicile comme acteur du système de santé aboutit à une complexité, et une instabilité, des dispositifs de financements. Elle rend également soutenable le 
désengagement de l'ordre politique dans la santé, la prise en charge de certaines fonctions relevant autrefois du champ de l'assurance maladie obligatoire étant transférées dans un champ de la dépendance auquel le non-rattachement à la santé a bien été institutionnellement signifié, et ceci même si le care doit être inscrit dans les structures productives du système de santé.

\section{L'aide à domicile dans les services à la personne : une relation ambiguë au marché}

Si l'aide à domicile n'est pas rattachée explicitement au système de santé, cette activité est en revanche attachée à l'entité des services à la personne (SAP). Constitués légalement par le Plan Borloo, dont la vocation première est la création d'emplois, ce champ regroupe un ensemble d'activités de services aux logiques historiquement distinctes, comprenant essentiellement des services de ménage/ repassage, des services d'aide à domicile à des personnes dépendantes. Ces activités sont unifiées par des mesures fiscales venant subventionner la demande (en particulier, une réduction d'impôt sur le revenu à hauteur de $50 \%$ des sommes engagées), dont l'objectif est de soutenir l'émergence d'un marché. Dans la même optique, le Plan Borloo s'est attaché à rendre contestable ce qui doit devenir le marché des services à la personne en levant ce qui avait été identifié comme des barrières à l'entrée. L'idée de l'ordre politique est de sortir les activités de services à la personne de leur autoproduction dans l'ordre familial pour qu'elles rejoignent l'ordre économique. Si la perspective est celle d'un marché autonome (dans l'ordre économique strict), la combinaison d'une action publique en faveur de la concurrence des prestataires et d'un subventionnement de la demande renvoie à ce qui est usuellement considéré comme un quasi-marché, qui trouve sa source dans l'ordre politique.

Potentiellement, plusieurs modalités d'articulations des trois ordres de B. Théret, impliquant autant de dispositifs d'allocation des ressources, sont donc en œuvre dans l'espace de régulation des SAP. Dans la mesure où ces dispositifs renvoient à des logiques différentes de consommation et de formation des prix, notre objectif de caractérisation de la confusion dans la régulation de l'aide à domicile appelle à les distinguer. Le propos de cette section est à la fois de montrer et de clarifier l'enchevêtrement entre ces logiques marchandes et quasi marchandes. À cet effet, nous cherchons à préciser les dimensions caractéristiques d'un quasi-marché relativement à un marché (2.1). Nous identifions trois dimensions dont nous interrogeons la présence au sein du secteur des services à la personne (2.2). Un résultat intermédiaire se dégage : parmi l'ensemble des activités de SAP, seuls les services de care présentent les trois dimensions d'une organisation quasi marchande, les autres services relevant d'une organisation marchande. Cette coexistence, au sein d'un même secteur, de marché et de quasi-marché (donc de deux formes pures de modalités d'articulation des ordres) nous conduira à interroger également la présence conjointe de ces dispositifs pour l'allocation des ressources d'un même type de service, l'aide à domicile auprès de personnes âgées (2.3). 


\subsection{Fonder une opposition entre marché et quasi-marché}

40 Si le quasi-marché est usuellement défini par la présence conjointe de financements publics et de prestataires en concurrence, cette conception ne semble pas suffisante dans l'espace des services à la personne et appelle précisions.

41 Le concept de quasi-marché a initialement été développé par Williamson pour désigner une forme intermédiaire de coordination des acteurs entre le marché (parfait) et la hiérarchie associée aux administrations. Il s'est progressivement imposé pour désigner une forme particulière de l'organisation de la protection sociale incarnée par les réformes du National Health Service au début des années 1990 et est désormais considéré comme un instrument de la mise en œuvre d'une nouvelle gestion publique opposée à l'économie planifiée . Usuellement, il est défini de manière inductive à partir du financeur et des principes de concurrence entre les opérateurs. La définition qui fait référence est celle de Le Grand qui ne parvient à le définir que dans une optique dynamique, où cette forme de coordination s'oppose à celles associées à une régulation tutélaire : « the intention is for the state to stop being both the funder and the provider of services. Instead it is to become primarily a funder, purchasing services from a variety of private, voluntary and public providers, all operating in competition with one other » (p. 1257).

42 La mise en œuvre d'une organisation de services sociaux par le quasi-marché se fait dans une optique d'efficience de l'utilisation des ressources publiques. L'idée sous-jacente est qu'un monopole public crée un gaspillage. Il est inefficient car il n'y a pas d'incitations à réaliser du profit, notamment de par l'absence de concurrents . L'organisation par la puissance publique d'une concurrence entre producteurs doit permettre de lever cet écueil.

43 Ce qui nous semble essentiel, bien qu'étant souvent implicite dans les travaux ayant trait à la mise en œuvre de quasi-marchés, est l'opposition de cette forme institutionnelle à une économie planifiée impliquant la production de services sociaux par l'État. Ainsi I. Greener et M. Powell pointent que l'introduction d'organisations quasi marchandes peut être justifiée par la présence d'externalités dans la production du service. Et, si les externalités ne sont pas intégrées dans la prise de décision individuelle, les bénéfices collectifs qui en résultent peuvent excéder les coûts. Sous cette condition, l'octroi de financements publics devient légitime. De la même manière, R. Flynn pointe la responsabilité de la puissance publique en termes de financement pour ce qu'elle organise par le biais de quasi-marchés: «The term quasi-market is used to underline the fact that the state still retains responsibility for financing services (through collective taxation and insurance contributions) and provision is basically linked to entitlement, with eligibility determined by administratively or professionally defined need, rather than ability to pay » .

L'introduction d'une dimension d'utilité collective aux services attachés au quasi-marché, constituant la justification du financement public, permet de préciser la caractérisation de la forme institutionnelle du quasi-marché . Celui-ci se définit alors à partir de trois dimensions :

- les quasi-marchés constituent une modalité d'allocation des services sociaux, ceux-ci présentant une dimension de bien commun, et ont une utilité collective ou quasi-collective qui en justifie le financement par la puissance publique ; 
- cette forme d'organisation implique une séparation entre l'acheteur, constitué par la puissance publique, et le producteur, qui de facto contractualisent l'achat d'une prestation de service ;

- les producteurs sont dans une situation de concurrence en vue de la contractualisation avec l'acheteur public, celui-ci pouvant agir directement ou par le biais des usagers du service dont la demande est alors fonction de leurs besoins exprimés et validés par la puissance publique (fonction d'évaluation) et non pas de leurs seuls revenus.

La présence conjointe de ces dimensions renvoie à un quasi-marché, associé à un régime de consommation relativement indépendant du revenu direct individuel alors que la consommation dans le cadre du marché est fonction du revenu (au sens large de revenu net d'impôts).

\subsection{Le quasi-marché : dispositif commun à l'ensemble des SAP?}

Les services à la personne comprennent des activités de care, parmi lesquelles se trouvent les SAD et des services que les concepteurs du Plan Borloo qualifient eux-mêmes de services de confort. L'ensemble des activités de services à la personne bénéficie de mesures de subventionnement (fiscal) du consommateur par la puissance publique. Pour toutes ces activités, un grand nombre de producteurs sont en concurrence, ces derniers pouvant être des organismes prestataires (associations ou entreprises), mais aussi des salariés directement employés par le bénéficiaire du service. Les dimensions de concurrence et de financements publics du quasi-marché y sont donc présentes. Mais les services à la personne sont-ils tous des services présentant une dimension d'utilité collective?

Les services de care auprès de personnes âgées ou handicapées et les services de garde d'enfants bénéficient de financements ciblés issus de la protection sociale. Les allocations sont déterminées à partir d'évaluations objectivées des besoins. En outre, dans la mesure où ces services, soit contribuent à la croissance démographique du pays, soit relèvent de la prise en charge de la santé des personnes âgées et/ou handicapées, ils peuvent être considérés comme des services sociaux qui présentent une dimension d'utilité collective, comme le suggèrent J-L Laville et $\mathrm{M}$. Nyssens en les qualifiant de bien (quasi)collectifs, entendus comme des services dont la consommation n'est pas strictement divisible. Ils attribuent ainsi à l'aide à domicile aux personnes âgées des effets en termes de santé publique et de cohésion sociale. M. Nyssens et F. Petrella qualifient également les services de care comme services quasi-collectifs, c'est à dire des services individuels mais qui sont également source de bénéfices pour la collectivité dans son ensemble, les bénéfices collectifs étant eux indivisibles. Enfin, l'idée d'un cinquième risque (cf. supra) réfère également à une dimension collective (cf. Annexe A).

Cependant, donner un caractère collectif ou quasi-collectif aux services de confort, de domesticité, pose problème. En effet, les services domestiques ne relèvent pas originellement de services sociaux, mais sont consommés par une classe supérieure disposant d'un revenu lui permettant de se décharger des tâches ménagères. Inclus depuis les années 1990 dans une politique d'emploi, les services domestiques peuvent néanmoins être positionnés à la frontière des services sociaux et de l'intérêt général : ils permettent de défamilialiser l'entretien du logement et agissent sur l'emploi, d'une part, en libérant les femmes de ces tâches et d'autre part en les faisant réaliser dans un cadre salarial par un tiers, cible de la politique d'emploi. Néanmoins, ces services ont un 
support matériel, le logement, support à partir duquel il est possible de préciser le consommateur du service comme étant la ou les personne(s) ayant l'usage du support. Ces services deviennent purement matériels et présentent davantage un caractère individuel que collectif, comme le suggèrent $M$. Nyssens et J-L Laville lorsqu'ils montrent le glissement des services de proximité, par nature quasi-collective, vers des services matériels ${ }^{14}$. Dans cette optique, à partir du moment où il est possible d'en distinguer le consommateur du service, les services de confort ne peuvent donc être considérés comme des services sociaux, présentant une dimension collective.

L'intervention de l'État en termes de subventionnement à la consommation peut également être interprétée comme une mesure de soutien à l'emploi, où l'emploi est subventionné et non le service consommé individuellement. Le service social serait alors l'emploi créé, ce qui augmenterait l'utilité collective. Mais si l'on peut effectivement considérer que les emplois créés par le recours à des services domestiques ont une utilité sociale, il faudrait alors qualifier ces emplois de façon suffisamment homogène pour être en mesure de désigner le service réellement produit. Or, les conditions d'emploi sont fortement hétérogènes dans le secteur aussi bien en termes de durée que de contenu de l'emploi, de rémunération que de nature de l'employeur... ${ }^{15}$. Cela suggère de ramener l'emploi à sa qualification la plus simple : un échange de travail organisé dans un cadre contractuel entre employeur et salarié.

Une telle qualification n'apparaît encore pas satisfaisante pour considérer que les services de confort sont organisés par un quasi-marché : l'accès au service social (emploi) se verrait conditionné par des critères ne relevant pas de la puissance publique, mais de l'employeur (contrairement aux Contrats d'insertion où le salarié doit remplir certaines conditions). En outre, au regard de la troisième dimension que nous avons attribuée au quasi-marché, la logique quasi marchande voudrait que ce soient les producteurs (soit, dans le cas où le service social est l'emploi, les consommateurs de services de confort qui produisent indirectement de l'emploi) et non les consommateurs (le salarié) qui soient mis en concurrence afin de bénéficier de financements publics.

51 Il ressort que les services domestiques (et plus largement les services de confort) ne relèvent donc pas d'une organisation quasi marchande bien que la puissance publique soutienne leur développement par un subventionnement du consommateur. Ce subventionnement, comme pointé par le Plan de développement des services à la personne, renvoie à une politique de soutien à l'émergence d'un nouveau secteur intensif en main-d'œuvre dans une logique industrielle de réorganisation sectorielle de la production. Il y a alors une juxtaposition voire dans certains cas une superposition de dispositifs au sein de ce qui est supposé constituer un même secteur: un marché subventionné pour les services à la personne et des quasi-marchés bénéficiant par ailleurs indirectement de mesures de soutien au marché.

Cela se traduit par deux logiques de consommation : l'introduction d'allocations pour les personnes âgées leur a permis de recourir à des services d'aide de façon autonome relativement à leurs revenus. À l'opposé, le recours à des services à la personne par des personnes d'âge actif (dont on peut alors supposer qu'elles ne recourent pas à des services d'aide à domicile) dépend grandement du revenu, les ménages les plus aisés ayant les plus forts taux de recours, mais aussi le plus fort levier de mobilisation des avantages fiscaux liés au marché. Sur ce type de service, le revenu constitue de fait un facteur déterminant de la demande. 

domicile comme instrument de politique d'emploi au même titre que les services de domesticité. L'aide à domicile bénéficie, au même titre que les services de conforts, des mesures fiscales destinées au décollage de l'ensemble du secteur. On a donc potentiellement une superposition de dispositifs de marché et de quasi-marché, ces dispositifs étant associés à des modalités différentes d'expression de la demande.

\subsection{Pour l'aide à domicile, une superposition de dimensions marchandes et quasi-marchandes}

Le cumul des objectifs d'emploi par la création d'un marché et de subventionnement de la demande de care conduit à rendre confus le mode d'allocation des ressources en vigueur, nécessitant de le préciser. On se propose donc d'interroger sur longue période la superposition des dispositifs introduits par les bricolages successifs en faveur de l'aide à domicile et la manière dont ils ont agi sur la demande d'aide.

\subsubsection{Mandataire et création de marché à enjeu quasi marchand}

Dans les années 1980, les associations font face à la contrainte d'une régulation tutélaire qui organise un rationnement de l'aide ne leur permettant pas de répondre aux besoins qu'elles ont identifiés . Profitant de dispositifs institutionnels favorables aux particuliers employeurs, elles ont imaginé le mode de production mandataire où une association propose un appui à un particulier dans ses fonctions d'employeur ${ }^{16}$. En s'appuyant sur des logiques issues de la domesticité, ce système a vocation à structurer une offre répondant à une demande exprimée d'aide à domicile. Son essor conduit à une modification des conditions d'allocation de l'aide à domicile ${ }^{17}$, qu'il est possible d'interpréter comme les prémices d'un phénomène de construction sociale d'un marché . Des modifications notables interviennent sur les deux dimensions majeures du marché que sont les prix et les quantités. La quantité d'aide dont pourra bénéficier la personne âgée ne dépend plus de l'évaluation objective des besoins réalisée par un tiers habilité ni des quotas disponibles, mais de la demande émise par la personne âgée, fonction de l'appréciation subjective de ses besoins sous contrainte de revenus. Ainsi, il n'est pas neutre que le mandataire soit apparu dans un contexte d'augmentation relative du revenu des personnes âgées. L'émergence du mandataire conduit également à des modifications substantielles sur la formation du prix final de la prestation payée par le bénéficiaire. Le mandataire cherche à diminuer les prix, relativement au prestataire, en externalisant une partie des coûts de production (coûts de structure) sur la personne âgée bénéficiaire des services et en profitant de la possibilité offerte aux particuliers-employeurs ayant besoin d'une tierce personne de bénéficier d'exonérations de cotisations sociales. Une partie des coûts de production est ainsi directement supportée par le client/employeur. D'autre part, dans le système tutélaire, le prix final dans les prestations d'aide ménagère des caisses de retraite dépend du revenu du bénéficiaire. Or, dans le cadre du mandataire, le prix final n'est plus seulement conditionné aux revenus du bénéficiaire, il dépend du salaire conventionnel en vigueur et des exonérations de cotisations dont bénéficie l'employeur, de la capacité éventuelle de négociation de l'intervenant pour son salaire, ainsi que des tarifs pratiqués par l'association mandataire. 


\subsubsection{Financement de la dépendance et superposition de dimensions marchandes et quasi-marchandes}

des associations qui proposent ces services comme instrument de politiques d'emploi. Ce positionnement de l'aide à domicile dans les politiques d'emplois s'est vu confirmé avec l'introduction de mesures de subventionnement de la demande visant à en favoriser l'essor, ainsi que celui d'autres services relevant des emplois familiaux, en particulier, dès 1991, des réductions d'impôts à hauteur de 50 \% des sommes engagées. Ces mesures de soutien à l'emploi seront réaffirmées en 2005 par le Plan Borloo. Elles se cumulent avec des mesures dédiées à la prise en charge de la dépendance: la Prestation spécifique dépendance (instaurée en $1997^{18}$ ) puis l'Allocation d'autonomie (APA en $2001^{19}$ ). Ces subventionnements directs sont dédiés à la réponse à la limitation induite par l'état de dépendance pour la réalisation des actes de la vie quotidienne (déplacements, hygiène, entretien du logement, ...). Leur montant est déterminé à la fois à partir du revenu du bénéficiaire et de la traduction monétaire d'une évaluation en volume des besoins réalisée par un représentant des pouvoirs publics (équipes médico-sociales des conseils généraux). L'introduction de ces allocations dépendance permet une certaine autonomisation de la demande par rapport à la contrainte budgétaire via un transfert de la contrainte budgétaire de la personne âgée à l'État. Ainsi, chez les personnes âgées, le recours à des services d'aide à domicile suit une courbe en $\mathrm{U}$ en fonction du revenu. Les recours les plus forts se situent, d'une part, parmi les tranches aux revenus les plus faibles et, d'autre part, parmi celles aux revenus les plus élevés. Les allocations d'autonomie sont donc bien caractéristiques de la première dimension que nous avons donnée au quasi-marché : elles financent des services sociaux, particulièrement pour les personnes les moins riches. Ce phénomène est renforcé et universalisé à partir du moment où l'on considère que le soutien public à la prise en charge à domicile de la dépendance se substitue à une prise en charge relevant du champ du soin, à domicile ou en établissement . Cette forme de prise en charge serait financée par la branche maladie de la Sécurité sociale, qui alloue des financements pour l'achat de services de soins de façon totalement indépendante du revenu du bénéficiaire.

In fine, la construction des services à la personne organise une imbrication originale d'un élément de la protection sociale organisé par un quasi-marché, les services de soutien à domicile, au sein d'un marché plus vaste dont la puissance publique souhaite (depuis 1991) soutenir le décollage. Or, les mesures fiscales destinées à soutenir l'émergence du secteur des services à la personne n'ont pas vocation à être pérennes et leur remise en cause pourrait venir se répercuter sur l'organisation quasi marchande du care et in fine sur les possibilités d'accès aux services sociaux de care.

\section{Conclusion}

Le positionnement analytique choisi pour caractériser la non-régulation de l'aide à domicile, au croisement du système de santé et de l'espace des services à la personne, nous permet de dégager deux résultats. En premier, de par son intégration aux services à la personne, l'aide à domicile présente concomitamment des dimensions marchandes (de l'ordre économique) et quasi-marchandes (de l'ordre politique). En second, l'aide à 
domicile participe à la régulation de la performance du système de santé tout en n'étant pas pleinement intégré à celui-ci.

Cela fait écho aux travaux de $B$. Théret lorsqu'il insiste sur l'irréductibilité de la sphère domestique, aux sphères économique (lieu de déploiement d'organisations économiques sous contraintes de ressources) ou politique (lieu de déploiement d'une organisation de la protection sociale), et sur les tensions entre ces sphères qui sont l'objet de la régulation. Le jeu de tensions, si l'on suit toujours $\mathrm{B}$. Théret ${ }^{20}$, trouve trois origines. D'une part, les « impasses économiques » d'un ordre politique, contraint de prendre en charge la montée en puissance de la problématique de la dépendance. D'autre part, dans la dimension politique de l'ordre économique, dans laquelle les acteurs, notamment associatifs ont été contraints de proposer une politique des organisations portant sur la façon de produire pour satisfaire les besoins observés en développant le mandataire. Et enfin, dans la prise en charge de l'articulation de l'ordre domestique avec ces deux ordres.

Dans l'ordre politique, nous observons la construction hésitante d'un espace de protection sociale (PSD et APA), simultanément à celle de dispositifs supports de relation de marché. Alors que dans l'ordre économique, les nécessités de la production du service font émerger des réseaux, les réseaux étant la seule alternative en termes de filière de santé cohérente à la constitution d'une filière de santé intégrée verticalement, qui ne peut se faire dans le contexte d'empilement institutionnel que nous avons décrit. Dans cet ordre sont donc reliés des espaces économiques structurés autour de régimes de qualité et de professionnalisation, ainsi que des régimes de prix de natures différentes.

61 Le paradoxe de cette situation est que la pluralité des dispositifs conduit à ce que ce soit dans l'ordre domestique que sont assemblées les ressources produites dans les deux autres ordres: c'est en effet à cet endroit que l'usager va combiner les différentes solutions offertes pour composer le service dont il a besoin. Les tensions dans le système d'allocation des ressources se répercutent alors sur les familles, qui en deviennent les "gestionnaires en dernière instance ». Cela explique qu'elles soient particulièrement impliquées sur les fonctions de surveillance et d'organisation de la coordination, mal prises en charge par les professionnels dans ce contexte où la régulation de l'aide à domicile manque de cohérence.

\section{BIBLIOGRAPHIE}

Allaire G., (2002), «L'économie de la qualité, en ses secteurs, ses territoires et ses mythes », Géographie Economie Société, 4 (2), pp. 155-180.

Béjean S. et M. Gadreau, (1997), « Concept de réseau et analyse des mutations récentes du système de santé », Revue d'économie industrielle, 81 (81), pp. 77-97.

Bressé S., (2003), « Les circuits de recours aux services d'aide domicile », Dossiers santé et solidarité, Les personnes âgées entre aide à domicile et établissement, 1, pp. 25-34. 
Buttard A. (2011), « Le réseau en santé : débat théoriques et enjeux politiques », dans $\mathrm{P}$. Batifoulier, A. Buttard et J.-P. Domin (éds), Santé et politiques sociales : entre efficacité et justice, Autour des travaux de Maryse Gadreau, Paris, Eska, pp. 131-143.

Cahuc P. et M. Debonneuil. « Productivité et emploi dans le tertiaire, » Paris : Conseil d'analyse économique 2004.

Cash E., R. Cash et C. Dupliet, (2011), « Étude sur la réactivité des établissements aux incitations tarifaires ", Document de travail de la DREES, série Etudes et Recherches, 106.

Claveranne J.-P., C. Pascal et D. Piovesan (2011), « T2A, vitesse et marchandisation », dans P. Batifoulier, A. Buttard et J.-P. Domin (éds), Santé et politiques sociales : entre efficacité et justice, Autour des travaux de Maryse Gadreau, Paris, Eska, pp. 247-259.

Colvez A., E. Cozette, A. Gramain et M.-E. Joël, (2000), « Situation économique et qualité de vie des aidants aux malades atteints de démence sénile de type Alzheimer », Revue économique, 51 (51), pp. 163-184.

Coriat B. et O. Weinstein, (2004), «Institutions, échanges et marchés », Revue d'économie industrielle, (107), pp. 37-62.

Coriat B. et O. Weinstein, (2005), « La construction sociale des marchés », La lettre de la régulation, 53, pp. 1-5.

Devetter F.-X., F. Horn et F. Jany-Catrice, (2011), « Interprétations localement différenciées d'une politique nationale : l'exemple des services à la personne ", Revue Française des Affaires sociales, 4, pp. 135-157.

Devetter F.-X., F. Jany-Catrice et T. Ribault (2008), Les services à la personne, Paris, La découverte.

Devetter F.-X. et S. Rousseau (2011), Du balai - Essai sur le ménage à domicile et le retour de la domesticité, Paris, Raisons d'agir.

Domin J.-P. (à paraître), Une histoire économique de l'hôpital ( $\mathrm{xx}^{e}$ siècle), Une analyse retrospective du développement hospitalier. Tome II (1945-2009), Paris, Comité d'histoire de la sécurité sociale, La documentation française.

Enjolras B., (1995), « Comment expliquer la présence d'organisations à but non lucratif dans une économie de marché ? : l'apport de la théorie économique », Revue française d'économie, pp. 37-66.

Enjolras B. et J.-L. Laville (2001), « France : des services locaux, associatifs et publics, confrontés à des règles nationales changeantes ", dans J.-L. Laville et M. Nyssens (éds), Les services sociaux entre associations, État et marché, L'aide aux personnes âgées, Paris, La découverte pp.

Fenina A., M.-A. Le Garrec et M. Koubi, (2011), « Les Comptes nationaux de la santé en 2010 », Études et résultats, 773.

Flynn R., (1997), "Quasi-welfare, associationalism and the social division of citizenship", Citizenship Studies, 1 (3), pp. 335-350.

Fraisse L. et L. Gardin (2012), « Les associations au coeur d'un enchevetrement de régulations », dans F. Petrella (éd), Aide à domicile et services à la personne : les associations dans la tourmente?, Rennes, Presses Universitaires de rennes, pp.

Gadreau M., (2001), « Les réseaux dans le système de santé et l'arbitrage efficacité-équité », Économie Appliquée, LIV (2), pp. 91-128.

Gallois F. «L'aide à domicile aux personnes âgées dépendantes au prisme du quasi-marché », Séminaire d'Economie Politique de la santé (Séposa). Paris 2012. 
Gallois F., (à paraître en 2013), « L'organisation publique de la concurrence est-elle source d'efficience ? L'aide à domicile », Revue Française de Socio-économie, 11.

Gallois F. (2010), « Le mandataire dans l'aide à domicile : émergence, diffusion et appropriation par l'État », dans F. Degrave, D. Desmette, E. Mangez, M. Nyssens et P. Reman (éds), Transformations et innovations économiques et sociales en Europe : Quelles sorties de crise ? Regards interdisciplinaires, Louvain, Presses Universitaires de Louvain, pp. 169-185.

Gallois F. (2012), «Le quasi-marché dans les services à la personne : pour une vision en trois dimensions ", dans N. Richez-Battesti, F. Petrella et P. Gianfaldoni (éds), Travail, organisations et politiques publiques : quelle « soutenabilité » à l'heure de la mondialisation?, Louvain, Presses universitaires de Louvain, pp. 335-350.

Gardin L., M. Nyssens et P. Minguzzi, (2010), «Les quasi-marchés dans l'aide à domicile : une mise en perspective européenne », Annals of Public and Cooperative Economics, 81 (4), pp. 509-536.

Greener I. et M. Powell, (2009), "The Other Le Grand? Evaluating the 'Other Invisible Hand' in Welfare Services in England”, Social Policy \& Administration, 43 (6), pp. 557-570.

Haut Conseil pour l'avenir de l'Assurance maladie. « Assurance maladie et perte d'autonomie » Paris : HCAAM, 2011.

Henry A., S. Nassaut, J. Defourny et M. Nyssens (2009), Économie plurielle et régulation publique. Le quasi-marché des titres-services en Belgique, Gand, Academia Press.

Jeandet-Mengual E. et J.-B. de Reboul. « Analyse et modalités de régulation de l'offre globale en soins infirmiers, note de synthèse réalisée par l'IGAS, » Paris : IGAS, 2008.

Kranklader E., (2012), «Évolution de l'offre hospitalière entre 2001 et 2009 : technicité croissante pour des séjours plus courts », Dossier Solidarité Santé, DREES, 25.

Laville J.-L. et M. Nyssens, (2000), “Solidarity-Based Third Sector Organizations in the "Proximity Services" Field: A European Francophone Perspective", Voluntas: International Journal of Voluntary and Nonprofit Organizations, 11 (1), pp. 67-84.

Le Grand J., (1991), “Quasi-Markets and Social Policy”, The Economic Journal, 101 (408), pp. 1256-1267.

Marbot C. (2008), « En France, qui recourt aux services à domicile? », France, portrait social édition 2008, Paris, INSEE, pp. 143-162.

Merrien F.-X., (1999), « La Nouvelle Gestion publique : un concept mythique », Lien social et Politiques, 41, pp. 95-103.

Newhouse J. P., (2003), “Reimbursing for Health Care Services”, Économie publique/Public economics ,2003/2, pp. en ligne.

Noguès H. (2011), « Régulation publique et accompagnement du vieillissement : la chronique des barres parallèles », dans P. Batifoulier, A. Buttard et J.-P. Domin (éds), Santé et politiques sociales : entre efficacité et justice, Autour des travaux de Maryse Gadreau, Paris, Eska, pp. 56-69.

Nyssens M. et F. Petrella, (2009), « Finalité sociale et partenariats public-privé dans l'offre de services quasi-collectifs locaux : une forme innovante de propriété », Economie et Société, série EGS, 10 (4), pp. 747-774.

OMS. « Préambule de la constitution de l'OMS, » 1946.

Or Z. et T. Renaud, (2009), « Principes et enjeux de la tarification à l'activité à l'hôpital (T2A) Enseignements de la théorie économique et des expériences étrangères », Working paper IRDES, DT23. 
Petrella F. (2012), Aide à domicile et services à la personne : les associations dans la tourmente ?, Rennes, Presses Universitaires de Rennes.

Raymond M., B. Roussille et H. Strohl. « Enquête sur les conditions de la qualité des services d'aide à domicile pour les personnes âgées, » Paris : IGAS, 2009.

Rouget S., (2012), « Quand l'hôpital doit faire recette : "la T2A" " , Archives de Pédiatrie, 19 (6, Supplement 1), pp. H304-H305.

Sentilhes-Monkam A., (2005), « Retrospective de l'hospitalisation à domicile, l'histoire d'un paradoxe », Revue Française des Affaires sociales, 2005/3 (3), pp. 157-182.

Shleifer A., (1985), “A Theory of Yardstick Competition”, The RAND Journal of Economics, 16 (3), pp. 319-327.

Théret B., (1995), « De la comparabilité des systèmes nationaux de protection sociale dans les sociétés salariales. Essai d'analyse structurale », Recherche et Régulation, Working, 1995-1.

Théret B., (1996), «Les structures élémentaires de la protection sociale », Revue Française des Affaires sociales, 4, pp. 165-188.

Théret B. (1992), Régimes économiques de l'ordre politique, Paris, Presses universitaires de France.

UNIOPSS, (2001), « Document conjoncturel, Budgets prévisionnels 2002 », Union Sociale, 150.

UNIOPSS, (2002), « Document conjoncturel, Budgets prévisionnels 2003 », Union Sociale, 160.

Uniopss (1985), Les alternatives à l'hospitalisation, Pour des réponses sociales et médico-sociales, Paris, Uniopss.

Uniopss. « Les associations face aux nouveaux dispositifs d'aide à domicile, » Paris : rapport pour le Ministère des Affaires Sociales, 1989.

Weber F. (2011), Handicap et dépendance, Drames humains, enjeux politiques, Paris, Editions rue d'Ulm.

Williamson O. (1975), Markets and Hierarchies, Analysis and Antitrust Implications, New York, Macmillan.

\section{ANNEXES}

\section{Annexe A - Caractéristiques de l'allocation des ressources par type de services à la personne}

\begin{tabular}{|l|l|l|}
\hline & Services domestiques / de confort & $\begin{array}{l}\text { Services personnes âgées relevant } \\
\text { de l'APA ou de la CARSAT }\end{array}$ \\
\hline $\begin{array}{l}\text { Financement } \\
\text { public du service }\end{array}$ & Impôt & Impôt et vouchers \\
\hline
\end{tabular}




\begin{tabular}{|l|l|l|}
\hline $\begin{array}{l}\text { Modalités } \\
\text { d'attribution du } \\
\text { financement }\end{array}$ & $\begin{array}{l}\text { Fonction croissante du revenu } \\
\text { (cette dimension a été tardivement } \\
\text { peutralisée par l'instauration de } \\
\text { concernent cependant que les seuls } \\
\text { couples biactifs) }\end{array}$ & $\begin{array}{l}\text { Fonction croissante du niveau de } \\
\text { dépendance (évaluation objectivée du } \\
\text { besoin par la collectivité) } \\
\text { Fonction croissante du revenu pour } \\
\text { les réductions d'impôt sur le revenu, } \\
\text { mais décroissante du revenu pour le } \\
\text { reste à charge sur les allocations } \\
\text { publiques }\end{array}$ \\
\hline $\begin{array}{l}\text { Principe } \\
\text { concurrence }\end{array}$ & $\begin{array}{l}\text { Marché subventionné à perspective } \\
\text { De marché autonome }\end{array}$ & $\begin{array}{l}\text { Quasi-marché(excluant } \\
\text { direct pour les allocations CARSAT) }\end{array}$ \\
\hline $\begin{array}{l}\text { Dimension } \\
\text { d'intérêt général }\end{array}$ & Emploi? & Emploi \\
Santé publique \\
Défamilialisation du care \\
Risque social
\end{tabular}

Source : réalisé par nos soins

\section{NOTES}

1. «Un espace de régulation est défini par deux dimensions : une structure productive (un système d'échanges ou transactions) et un ordre institutionnel (règles) » . L'espace de régulation ne correspond pas nécessairement à un mode de régulation complet, ce qui permet d'interroger la cohérence entre les différents espaces de régulation.

2. Parmi les vingt-et-une activités de services à la personne, définies par le décret 2007-854 du 14 mai 2007 relatif aux services à la personne, on trouve également des services de dépannage informatique, de soutien scolaire, de bricolage et jardinage...

3. Le budget de l'hôpital, dans ce système de tarification, dépend du prix de journée établi et du nombre de lit et du taux d'occupation de ces lits.

4. Caisse Régionale d'Assurance Maladie.

5. Source : entretiens semi-directifs menés avec plusieurs dirigeants d'associations et entreprises d'aide à domicile de la région de Reims, essentiellement au printemps 2011.

6. Les données Eco-santé (issues de sources DREES) indiquent un taux d'équipement de 55,7 places pour 1000 habitants de plus de 75 ans en 2001, de 18,2 en 2002 et de 44,7 en 2003.

7. Arrêté du 25 mai 1973 relatif à l'exonération des cotisations patronales afférentes à l'emploi d'une tierce personne.

8. Loi 75-534 du 30 juin 1975 d'orientation en faveur des personnes handicapées.

9. L'ACTP est une prestation d'aide sociale qui est gérée et versée par les conseils généraux. Elle concerne des personnes ayant un taux d'incapacité reconnue d'au moins $80 \%$ et nécessitant une aide à la vie quotidienne. Elle est soumise à une condition de ressources (et depuis qu'existent des prestations spécifiques pour personnes âgées, elle est également conditionnée à un critère d'âge, la personne devant avoir moins de 60 ans lors de la demande). Le montant de l'allocation est fonction des besoins et de la façon dont ils sont pris en charge.

10. Le développement $d u$ reste à charge et de l'assurance maladie complémentaire tend cependant à introduire un tel type de discrimination. 
11. La Loi du 2 janvier 2002 rénovant l'action sociale et médico-sociale redéfinit le champ de l'action sociale et médico-sociale, organise les relations entre les établissements autorisés et leur tutelles, notamment pour ce qui est de l'autorisation médico-sociale et de la tarification; elle instaure également un ensemble d'instruments destinés à affirmer les droits des usagers comme par exemple le conseil de la vie sociale.

12. Cette situation de sous-tarification aboutit à une crise des organismes autorisés d'aide à domicile, pour un analyse plus large de la situation critique de l'aide à domicile et de ses causes, le lecteur pourra se référer au récent ouvrage coordonné par F. Petrella .

13. Loi $n^{\circ} 2009-879$ du 21 juillet 2009 portant réforme de l'hôpital et relative aux patients, à la santé et aux territoires.

14. " evidence shows that valued services with a high subjective proximity context have been displaced by more "material" activities. For example, recent evaluations of ALE have shown that the majority of activities on this scheme tend to benefit users' families, including household work or small gardens, rather than relational social care activities, such as home care for sick children " .

15. La politique belge des Titres-services autorise une meilleure homogénéisation des emplois qu'elle cherche à créer. L'employeur y est nécessairement un organisme ayant obtenu un agrément, cet agrément précise que l'organisme doit, sous certaines conditions d'ancienneté, offrir des CDI à ses travailleurs, que les anciens allocataires sociaux sont prioritaires pour obtenir des emplois à temps plein. Par ailleurs la mise en œuvre par les employeurs de formation des personnes devant être insérées par le dispositif est évaluée. Enfin, le dispositif Titre-service est pensé pour les seuls services ménagers (et non les services de care), ce qui, au point de vue formel, permet « d'uniformiser » les activités des travailleurs .

16. Ayant émergé dès le début des années 1980, le mandataire sera institutionnellement reconnu, par le biais d'une circulaire, en 1987. Sur l'émergence du mandataire, le lecteur pourra se référer à Gallois .

17. Une telle marchandisation des services apparaît également si l'on considère le recours à des services prestataire d'aide ménagère hors du cadre de régulation organisé par les prestations des caisses de retraite.

18. Loi $n^{\circ} 97-60$ du 24 janvier 1997 tendant, dans l'attente du vote de la loi instituant une prestation d'autonomie pour les personnes âgées dépendantes, à mieux répondre aux besoins des personnes âgées par l'institution d'une prestation spécifique dépendance.

19. Loi $\mathrm{n}^{\circ}$ 2001-647 du 20 juillet 2001 relative à la prise en charge de la perte d'autonomie des personnes âgées et à l'allocation personnalisée d'autonomie

20. Cette fois dans son ouvrage sur les régimes économiques de l'État

\section{RÉSUMÉS}

Cet article examine la régulation de l'aide à domicile auprès de personnes âgées dépendantes à travers deux espaces possibles, mais aux logiques différentes: celui de la santé et celui des services à la personne. Il montre en premier lieu que, si l'aide à domicile constitue un support de la performance du champ du soin, cette activité reste à la frontière du système, à la fois nécessaire et exclue. En second, l'aide à domicile est examinée en tant que cible d'une politique d'emploi mise en œuvre au moyen d'un soutien public à l'émergence d'un marché des services à la personne. Il se dégage que, si l'aide à domicile est effectivement organisée en partie par une 
logique marchande, la logique quasi-marchande (incluant un lien avec la protection sociale) semble dominante. Au global, on retient davantage une non-régulation de l'aide à domicile qu'une régulation.

This paper examines the regulation of domiciliary care services (DCS) for elderly people throughout two different areas of regulation: the health system and the institutional sector of "lifestyle and domiciliary care services". DCS potentially belong to these two areas, but both refer to different logics. The first result shows that even if the DCS support the performance of the field care in the health system, it stays at the border of the health system by being both necessary and excluded. The second result considers DCS as belonging to lifestyle and home cares services. All these services are being subjected to a particular employment policy developed by the French State while also being supported by State in order to allow the emergence of a new market. In terms of regulation, I underline that exchanges for DCS are partly relying on market, but they are mostly relying on quasi-market (and, consequently, Social State). These two results seem to indicate that DCS are essentially unregulated.

\section{INDEX}

Keywords : home care services, human resources management tools, territorial organizations network, normalisation

Mots-clés : aide à domicile, services à la personne, régulation sectorielle

Code JEL Q01 - Sustainable Development, A2 - Economic Education and Teaching of Economics, B52 - Institutional • Evolutionary

\section{AUTEUR}

\section{FLORENCE GALLOIS}

Laboratoire Regards, EA 6292 Florence.gallois[at]gmail.com 